
THE UNIVERSITY

OF ILLINOIS

LIBRARY

595.1

$92 e$

Blatogy

BHOAOY

oc 





$$
\text { IXNAIES }
$$

\section{SCIENCES NATURELLES.}

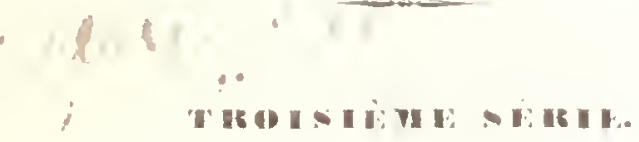

T,OOLOFIIE. 
Digitized by the Internet Archive $1 /$ M. : in 2011 with funding from University of Illinois Urbana-Champaign

http://www.archive.org/details/observationssurl310dure 
(IBSERYITIONG

SUR LES HEURES DU KÉVEIL ET DU CIMANT DE QUELQUES MISEAUX MLRVES

EV MAI ET JUIN 1846 ;

Par M. DUREAU DE IA maILE.

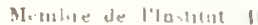

Ces observations ne portent que sur huit espèces d'oiseaux diurnes, qui habitent tous, pendant le printemps et l'été, le jardin de ma maison, rue de la Rochefoucault. Le calendrier du réveil et du chant de mes hôtes a été dressé charue nuit, ¿a Paris, depuis le $1^{\text {rr }}$ juin jusiguau 6 juillet 1846 , et dans ma terre de landres, près de Mortagne, département de l'Orne, depuis le 7 jusqu'au 23 juillet de la mème année.

Ces huit espices d'oiseaux sont, en les rangeant suivant l'ordre d'antériorité de leur réveil et de leur chant, depuis le $1^{\prime r}$ mai jusqu'au 6 juillet :

$1^{\circ}$ le P'inson, d'une heure it une heure et demie du matin;

$2^{\circ}$ La Fauvette à tète noire, de deux à trois heures:

$3^{\circ}$ Ia Caille, de deux heures et demie is trois heures (2));

$4^{\circ}$ Le Merle noir, de trois heures et demie à quatre heures:

$5^{\circ}$ Le Rossignol de murailles ou linurette à ventre rouge, de trois heures at trois heures et demie;

$6^{\circ}$ Le Pouillot, it quatre lueures ;

$7^{\circ}$ Le Hoineau franc, de cint heures in cinq houres et demie;

$S^{\circ}$ La Mésange charbonnière ou grosse Mlésange, de cinq hemres à cinq heures et demie.

On voit, par ces chiffres, que le Pinson est le plus matinal, et le lloineau le plus paresseux des oiseanx que j’ai observés. Est-ce de cette halbibde pomme qu'est venu le dicton : Gai, éreillé comme un Pinson? Muant au Moineau, qui vit dalls la so-

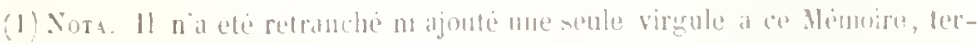
mine on 1896.

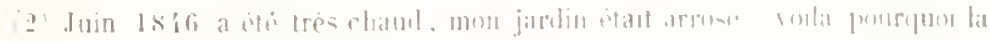

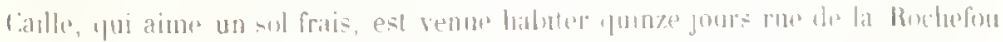

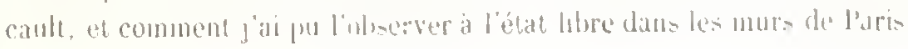




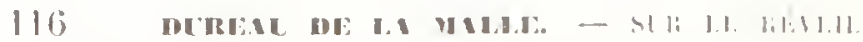

ciété de l'llomme, et qui pullule clims les villes. atmait-il contracté, par cette cohabilation, les haldilude - parensenses des olsilis

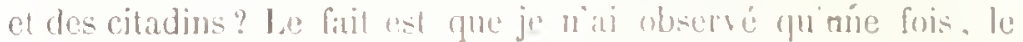

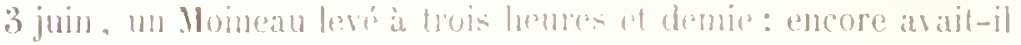
été éreille pale un Mere prise, habitant de ma matison. gui, depuis une heure. sithail à phein eronerer.

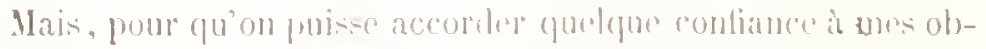

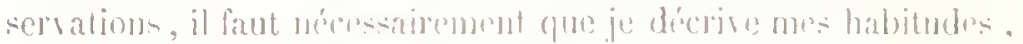

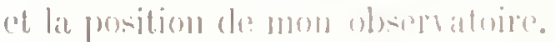

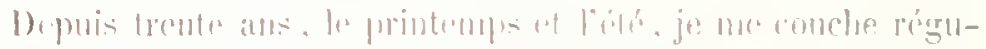

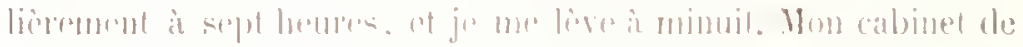

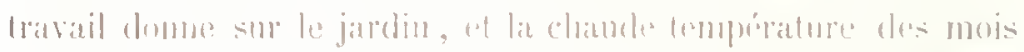

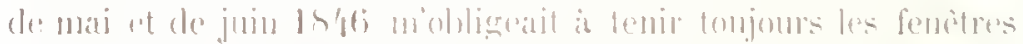

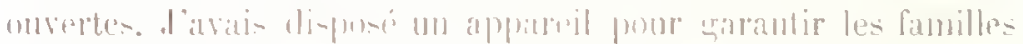

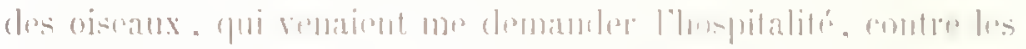

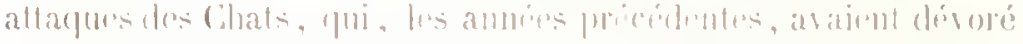

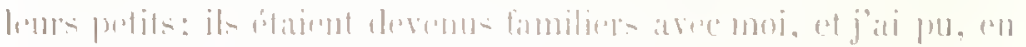

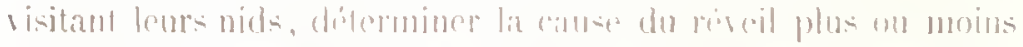

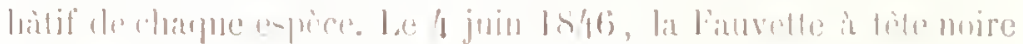

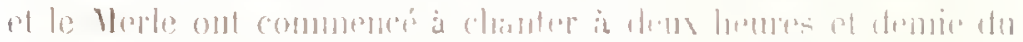

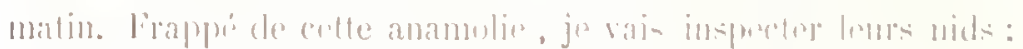

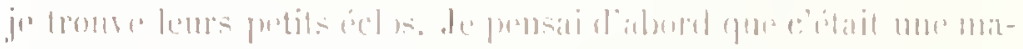

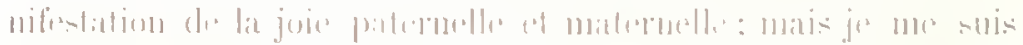

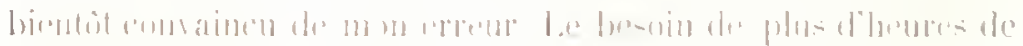

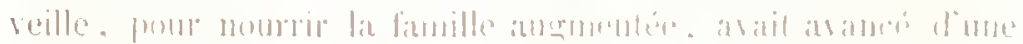

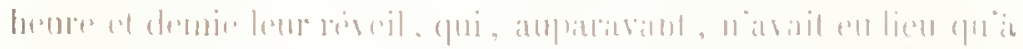

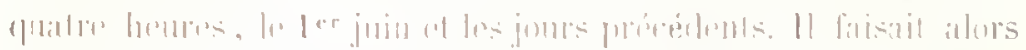

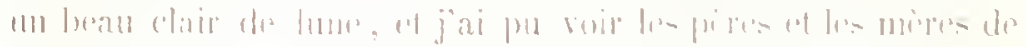

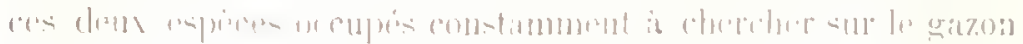

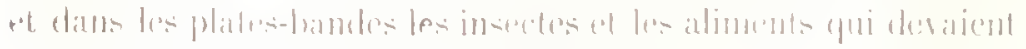

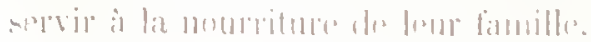

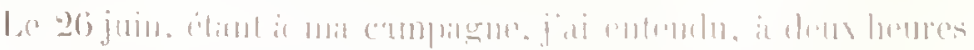

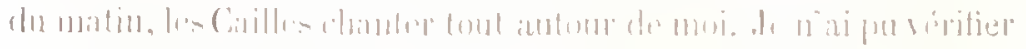

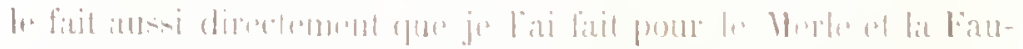

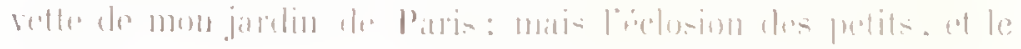

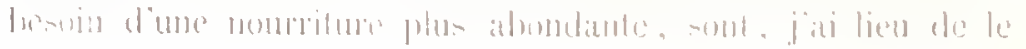


croire, la véritable canse de ce riveil anticipé, qui devance de deux henres te lever du soleil.

Je terminerai celte combe unte par une olservation qui prouve

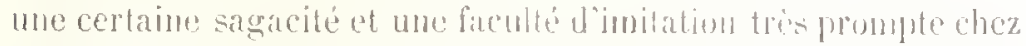
deux especes l'oiseaux chanteurs: la Jiancette is lite noire et le Jerle.

Le 11 juin, je nótais levé à misnuit, fes fenètres de nua bibliothèque vinvertes of ma lampe Cared allumer.

A minuit el demi, la Favete s'éveible, et chante sur l'Acacia platé a qualre metres de ma lenetre. J'rend-elle pour lo jour te Cilrcel qui belaire ma reille? I ne heure et demie se prase, elle

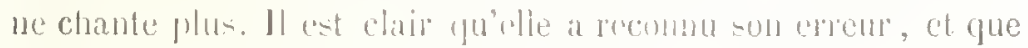
ce petit globe cullammé nest pas le suleil. Il est certain aussi que ce n'est pas le besoin d'une jlus grande quantité de momeriture

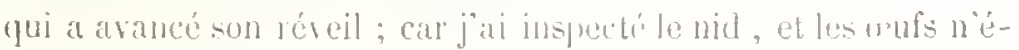
taient pas édos.

Yon portier nourrissail en cage un Merle furié, anyucl il avait

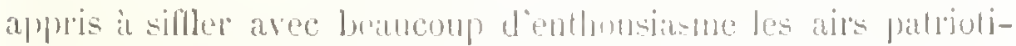
ques de la Marseillaise et the la C'urmatume. of qu'il plarait dans la cour, pris des fenètres de ma bibliothingur. On le rentermait tors les soirs dans unc chambre ubsecure. le s juin, un onblie de le rentrer; des minuit un quart, tronuré par l'éclat de ma lampe, il éreille toute la maimen en rhantant is gorge déplone les

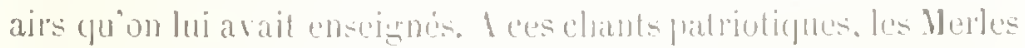
saurages répondent; et de minuit un quart it sept lecures du

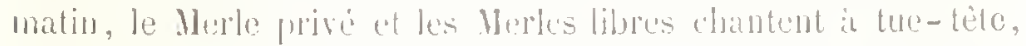

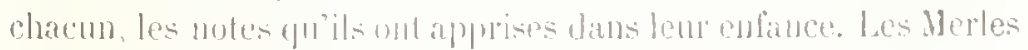
saurages stalient coltainement ontraines far un guide trumpeur ;

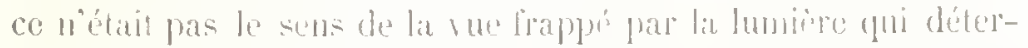
minait cette explosion musicale, car teur nid élait place is trente

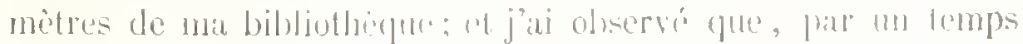

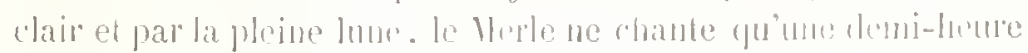

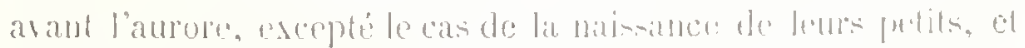

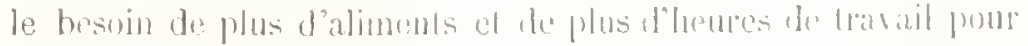
se procurer leur numriture.

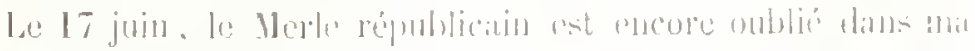

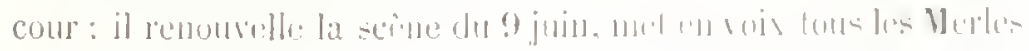


libres, of reseille de nourrau fous les labitants de ma maison. Je descinds, ot, pour le punir. je le mets au cachot dans un endroit obscur. Aubout dune heure, le crovant corrige, je le délive

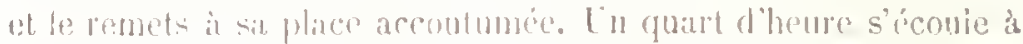

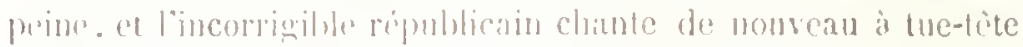

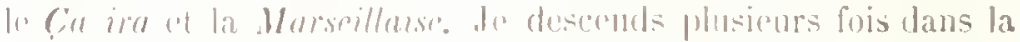

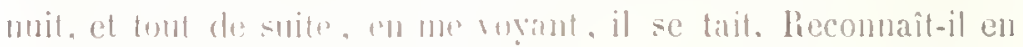
numi son juge? at sum greiliar?

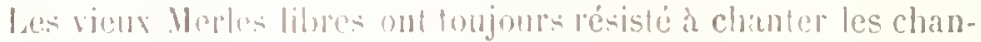

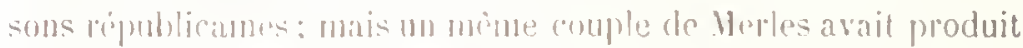

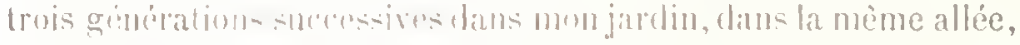

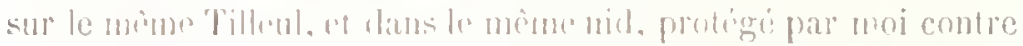

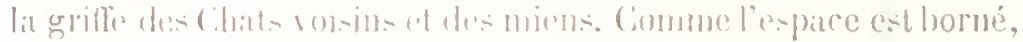

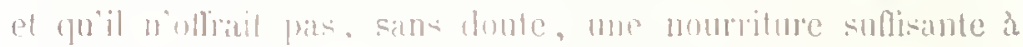

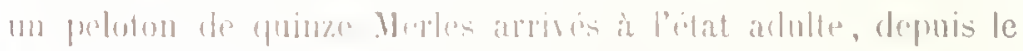

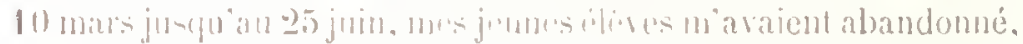

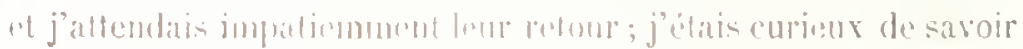

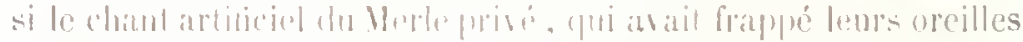

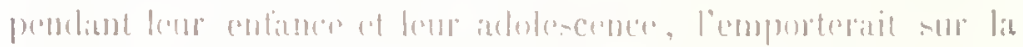

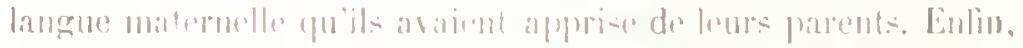

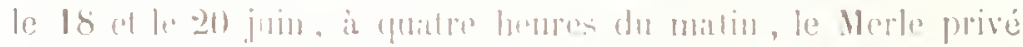

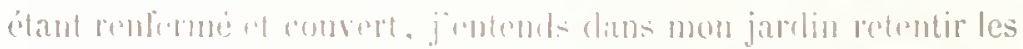

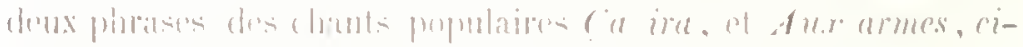

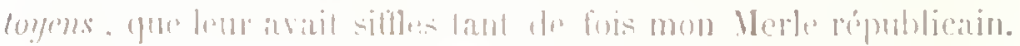

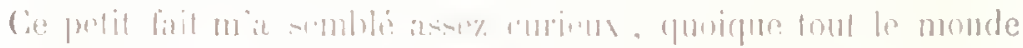

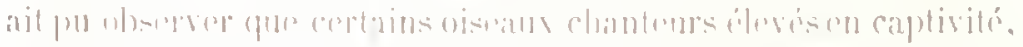

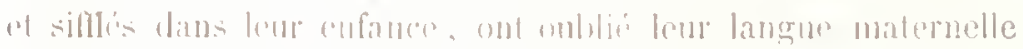

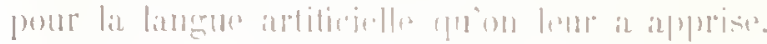

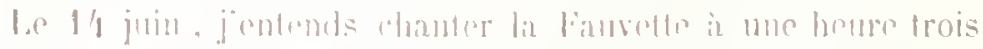

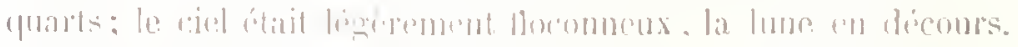

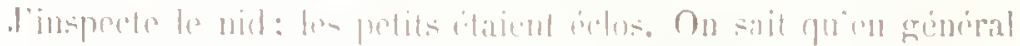

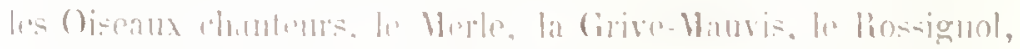

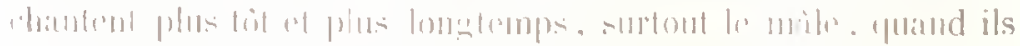

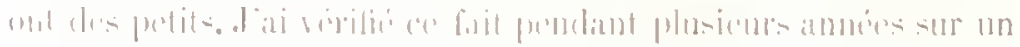

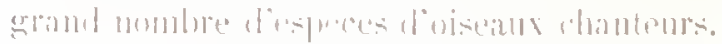

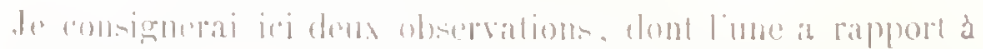

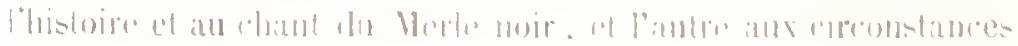


locales et encore inconnues qui penvent influer sur l'organe voeal.

Chatut du Merle nor d'tlitlit.

En mai et juin 1830 , j’ai traversé a picel les Apennins, de Vovi à Gênes el de Gènes à Carrare. J'ai sté fort supris d'entendre le Merle noir sauvage moduler un cluant tris me molicux tout diffórent de celui de cet oiscan dans nos contrés. Te me suis grlissé sans bruil, je me suis caché ; alors j’ai vu et ouï chanter l'oiseau à sept mètres de moi. l,e mâle surtout, au bec jaune, aux pieds noirs, au plumage noir sars lache, ne m'a lajssé nul doute sur l'identité de l'espèce. Ie clinat, les eaux, l'alir, les montagnes, le plus ou moins de lemsion électripue, ont-ils une intluence sum la voix? Bref, l'oiscan est le móme, son chant est Inut dillérent de celui de notre Merle. Les réfugiós italions, chasseurs et musiciens, onf éprouvé la même surprise que moi pour lo Merio italien, en entenlant chanter notre Mrel parision par une belle soirce, dans nos jardins de la rue de la Rochefuncanlt. 11. A. de llumboldt m'a assurí que la mème varialion existail dans les Canaries pour lo chant des Serius.

En 1811, jarais visilé Bergame. J'y remarquai ia la fois beancoup de goitres et des voix en grand nombre lres fortes et tris mélodieuses. Celte position, les rirconstances imennumes que j’ai indiquées plus haut ou d'autres encoro, intluent-elles en bien ou en mal sur l'organe vocal? Je consignai le fail. Srarpa et Mascagni avec qui j'en causai, ne dirent que le fait reconnu par eux était le nombre de goitreux et de bellos voix plus considérable à Bergame que dans aucune ville d'Italie, et hors de proportion avee les populations respertives.

Le professeur Orioli a aussi observé le mème fait, el m’a transnis son témoignage. le mème phénomène avait été dejjà obserwó à Ziuma par Vilruve uf Pline, témoins oculaires (1).

J'ai joint a ce petit Mémoire un ablean contenant les heurss du chant de huit espores d'Oisenux, avec les maxima el minima de temperiralure.

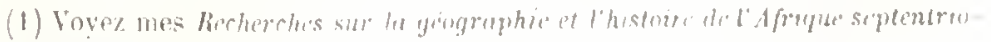
nule, arlicle $Z_{a} s a$ 


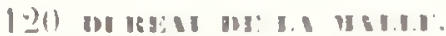

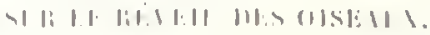

Tableau des heures du reveil de quelques enprees d'Oiseaus.

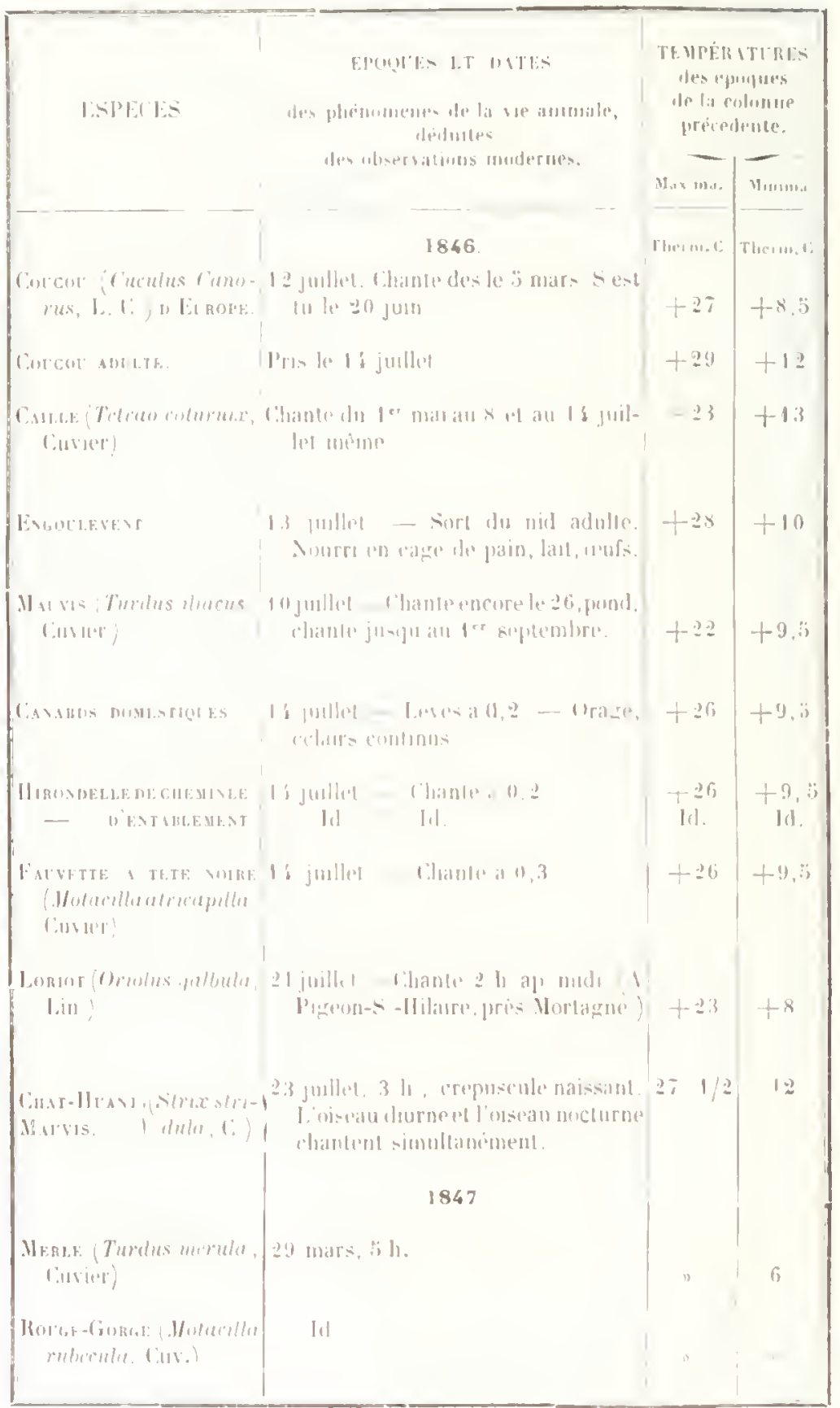




\section{TABLE DES MATEGES}

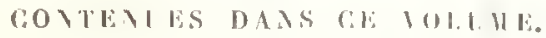

\section{ANATOYIE ET PIIVIMLOGIE.}

Sote sur le non-vomissement du Cheval par M Finnexs.

Mémoire pour servir à la connaissance de l'organisation a de la vie de la substance contractile thez les animaus les plun inlirieus; par M. ALE licher

\section{A NIMUX VERTERHLS.}

Observations sur les heurs du réveil alu danl dr yuelques Oiseaux

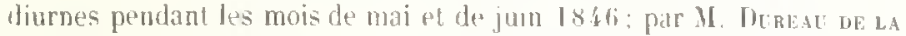
MALE.

Sur les animaux vertébrés envisagés sons lo loulle rapport de la géonraphie zoologigue el de la domestication ; par II Paur. Gervas.

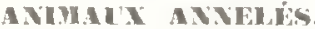

Cindes sur les types inférieurs de l'embranchement des Anneles. - Mémoire sur la famille des Hermelliens (Hermellen. Xols) : jatr. .I. I. ve Uitreages

Note sur quelques especes nouvelles du genor l'agner; far al Mave Enwards

Note sur les Coléopteres du gente Eurhinus, we lat fanille des Corruloniens: par M. Eume Blanchubd

Etudes embryogéniques. - Hémorre sur l embryogrine des Annelduter par M. A. de (UTMTrehages

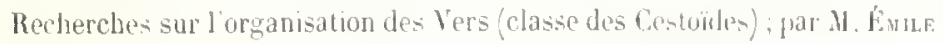
BLANCHARD

\section{MOLLengersi.}

Résumé des observations faites en IS 4' sur les Gastéropoles Plabentérés; par 11. . he Quatrefalies.

\section{Z(O)PIXTES.}

Recherches sur les polypiers. - Troisieme Mimoiri?: Honographio de.

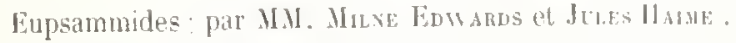

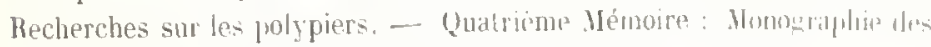

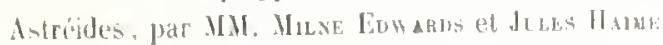


TABIE DES MUTIRES PMR MOUS D'MUTULS

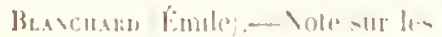
lolvoptegen du gemse fiuhmus,

- Recheredien sur lureanisilunis

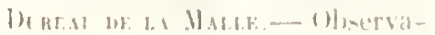

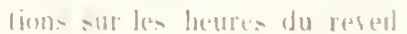
id duclant de quelques Dineaus diurnes prondant les mors de mai af de juin Isil

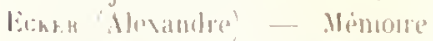

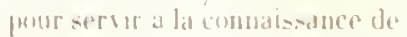

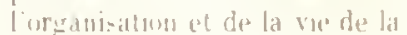

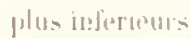

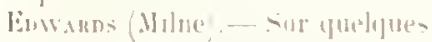

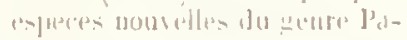
mure

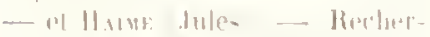

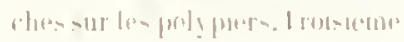

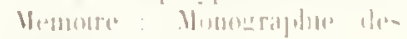

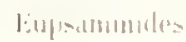
de la fanullo de- Lurculamiens. 1:3

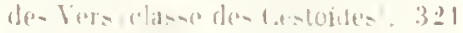

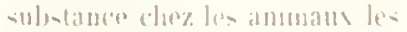

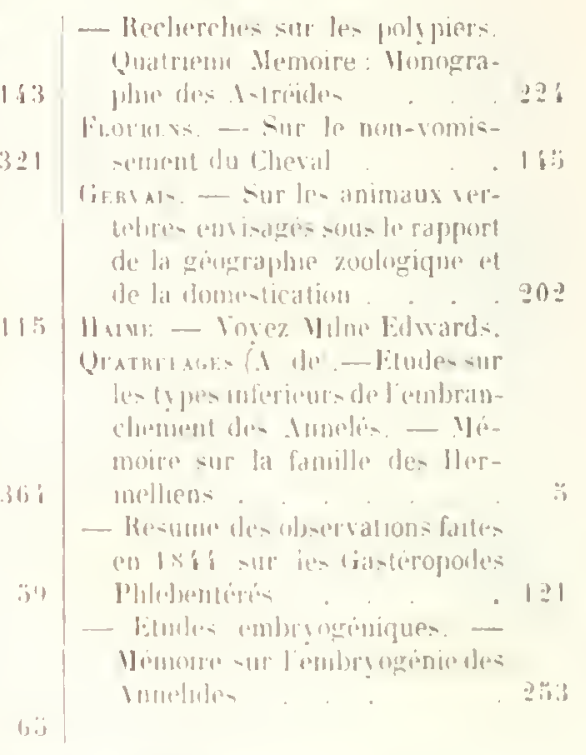

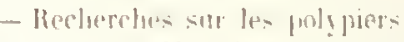
Uuatrusu Memoire: Honogra

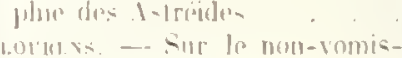
-rmont du Cluevil _. . I

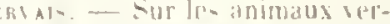

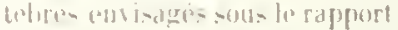
de la gencraplase zoologique et de lat domb-lication atritaters

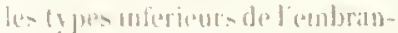

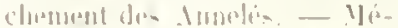
moire sur la famille den llor-

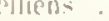
$;$

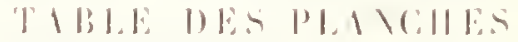

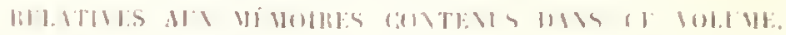

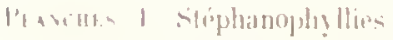

2. Mrganisation der. Hermellex.

3. Limbriogenice de- Hormallex

- Imbryegranie des Hermellem.

i). Eusmiliens.

(i) Eusmuliens.

- Lusulilina et Astróns

8 . Istremen.

9. Intréplix.

10 listmanac do lideral.

11 Mrganisation des Timias.

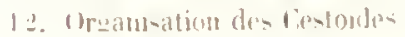

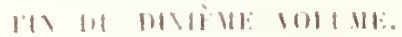




$$
\frac{77}{97}
$$










